\title{
A DETERMINANTAL EXPRESSION AND A RECURRENCE RELATION FOR THE EULER POLYNOMIALS
}

\section{FENG QI}

Institute of Mathematics, Henan Polytechnic University, Jiaozuo 454010, Henan Province, China; College of Mathematics, Inner Mongolia University for Nationalities, Tongliao 028043, Inner Mongolia Autonomous Region, China; Department of Mathematics, College of Science, Tianjin Polytechnic University, Tianjin 300387, China

\section{BAI-NI GUO}

School of Mathematics and Informatics, Henan Polytechnic University, Jiaozuo 454010, Henan Province, China

\begin{abstract}
In the paper, by a very simple approach, the author establishes an expression in terms of a lower Hessenberg determinant for the Euler polynomials. By the determinantal expression, the author finds a recurrence relation for the Euler polynomials. By the way, the author derives the corresponding expression and recurrence relation for the Euler numbers.
\end{abstract}

\section{MAin Results}

It is known that a matrix $H=\left(h_{i j}\right)_{n \times n}$ is called a lower (or an upper, respectively) Hessenberg matrix if $h_{i j}=0$ for all pairs $(i, j)$ such that $i+1<j$ (or $j+1<i$, respectively). Correspondingly, we can define a lower (or an upper, respectively) Hessenberg determinant.

It is general knowledge that the Bernoulli numbers and polynomials $B_{k}$ and $B_{k}(u)$ can be generated respectively by

$$
\frac{z}{e^{z}-1}=\sum_{k=0}^{\infty} B_{k} \frac{z^{k}}{k !}=1-\frac{z}{2}+\sum_{k=1}^{\infty} B_{2 k} \frac{z^{2 k}}{(2 k) !}
$$

E-mail addresses: qifeng618@gmail.com, qifeng618@hotmail.com, bai.ni.guo@gmail.com, bai.ni.guo@hotmail.com

2010 Mathematics Subject Classification. Primary 11B68, Secondary 11C20, 11Y55, 15A15, 26A06, 33B10, 65F 40 .

Key words and phrases. determinantal expression; recurrence relation; Euler polynomial; Euler number; Hessenberg determinant.

This paper was typeset using $\mathcal{A M}_{\mathcal{M}}$ - $\mathrm{LAT}_{\mathrm{E} X}$. 
and

$$
\frac{z e^{u z}}{e^{z}-1}=\sum_{k=0}^{\infty} B_{k}(u) \frac{z^{k}}{k !}
$$

for $|z|<2 \pi$. Because the function $\frac{x}{e^{x}-1}-1+\frac{x}{2}$ is odd in $x \in \mathbb{R}$, all of the Bernoulli numbers $B_{2 k+1}$ for $k \in \mathbb{N}$ equal 0 . The first six Bernoulli numbers $B_{2 k}$ for $-\leq k \leq 5$ are

$$
B_{0}=1, \quad B_{2}=\frac{1}{6}, \quad B_{4}=-\frac{1}{30}, \quad B_{6}=\frac{1}{42}, \quad B_{8}=-\frac{1}{30}, \quad B_{10}=\frac{5}{66} .
$$

In [4, Section 21.5] and [5, p. 1], it was listed that

$$
B_{k}=k !\left|\begin{array}{ccccccc}
1 & 0 & 0 & \cdots & 0 & 0 & 1 \\
\frac{1}{2 !} & 1 & 0 & \cdots & 0 & 0 & 0 \\
\frac{1}{3 !} & \frac{1}{2 !} & 1 & \cdots & 0 & 0 & 0 \\
\vdots & \vdots & \vdots & \ddots & \vdots & \vdots & \vdots \\
\frac{1}{(k-1) !} & \frac{1}{(k-2) !} & \frac{1}{(k-3) !} & \cdots & 1 & 0 & 0 \\
\frac{1}{k !} & \frac{1}{(k-1) !} & \frac{1}{(k-2) !} & \cdots & \frac{1}{2 !} & 1 & 0 \\
\frac{1}{(k+1) !} & \frac{1}{k !} & \frac{1}{(k-1) !} & \cdots & \frac{1}{3 !} & \frac{1}{2 !} & 0
\end{array}\right| .
$$

In [7, Theorem 1.2], the Bernoulli polynomials $B_{k}(u)$ for $k \in \mathbb{N}$ were expressed by a lower Hessenberg determinant

$$
B_{k}(u)=(-1)^{k}\left|\frac{1}{\ell+1}\left(\begin{array}{c}
\ell+1 \\
m
\end{array}\right)\left[(1-u)^{\ell-m+1}-(-u)^{\ell-m+1}\right]\right|_{1 \leq \ell \leq k, 0 \leq m \leq k-1}
$$

and, consequently, the Bernoulli numbers $B_{k}$ for $k \in \mathbb{N}$ were represented by a lower Hessenberg determinant

$$
B_{k}=(-1)^{k}\left|\frac{1}{\ell+1}\left(\begin{array}{c}
\ell+1 \\
m
\end{array}\right)\right|_{1 \leq \ell \leq k, 0 \leq m \leq k-1} .
$$

It is common knowledge that the Euler numbers $E_{k}$ and the Euler polynomials $E_{k}(x)$ can be generated respectively by

$$
\frac{2 e^{t / 2}}{e^{t}+1}=\sum_{k=0}^{\infty} \frac{E_{k}}{k !}\left(\frac{t}{2}\right)^{k}
$$

and

$$
\frac{2 e^{x t}}{e^{t}+1}=\sum_{k=0}^{\infty} \frac{E_{k}(x)}{k !} t^{k}
$$

which converge uniformly with respect to $t \in(-\pi, \pi)$. By these definitions, it is easy to see that

$$
E_{k}=2^{k} E_{k}\left(\frac{1}{2}\right)
$$

Since the generating function $\frac{2 e^{t / 2}}{e^{t}+1}$ of the Euler numbers $E_{k}$ is even on $(-\pi, \pi)$, then $E_{2 k-1}=0$ for all $k \in \mathbb{N}$. The first nine Euler numbers $E_{2 k}$ for $0 \leq k \leq 8$ are

$$
1, \quad-1, \quad 5, \quad-61, \quad 1385, \quad-50521, \quad 2702765, \quad-199360981
$$

and the first six Euler polynomials $E_{k}(x)$ for $0 \leq k \leq 5$ are

$$
1, \quad x-\frac{1}{2}, \quad x^{2}-x, \quad x^{3}-\frac{3}{2} x^{2}+\frac{1}{4}, \quad x^{4}-2 x^{3}+x, \quad x^{5}-\frac{5}{2} x^{4}+\frac{5}{2} x^{2}-\frac{1}{2} .
$$


At the website [9], the Euler numbers $E_{2 k}$ were represented by a lower Hessenberg determinant

$$
E_{2 k}=(-1)^{k}(2 k) !\left|\begin{array}{cccccc}
\frac{1}{2 !} & 1 & 0 & \cdots & 0 & 0 \\
\frac{1}{4 !} & \frac{1}{2 !} & 1 & \cdots & 0 & 0 \\
\vdots & \vdots & \vdots & \ddots & \vdots & \vdots \\
\frac{1}{(2 k-2) !} & \frac{1}{(2 k-4) !} & \frac{1}{(2 k-6) !} & \cdots & \frac{1}{2 !} & 1 \\
\frac{1}{(2 k) !} & \frac{1}{(2 k-2) !} & \frac{1}{(2 k-4) !} & \cdots & \frac{1}{4 !} & \frac{1}{2 !}
\end{array}\right|, \quad k \in \mathbb{N} .
$$

In [8, Theorem 1.1], the Euler numbers $E_{2 k}$ for $k \in \mathbb{N}$ were represented by a lower Hessenberg and sparse determinant

$$
E_{2 k}=(-1)^{k}\left|\left(\begin{array}{c}
i \\
j-1
\end{array}\right) \cos \left((i-j+1) \frac{\pi}{2}\right)\right|_{(2 k) \times(2 k)} .
$$

There have been many explicit expressions for the Euler numbers $B_{k}$ and the Euler polynomials $E_{k}(x)$. See, for example, the recently-published papers [2, 3, 8] and plenty of references therein.

In this paper, by a very simple approach, we will establish a new expression in terms of a lower Hessenberg determinant for the Euler polynomials $E_{k}(x)$. By the new determinantal expression, we will find a recurrence relation for the Euler polynomials. By the way, we will derive the corresponding expression and recurrence relation for the Euler numbers $E_{k}$.

Our main results can be summarized up as two theorems below.

Theorem 1. For $k \geq 0$, the Euler polynomials $E_{k}(x)$ can be expressed as

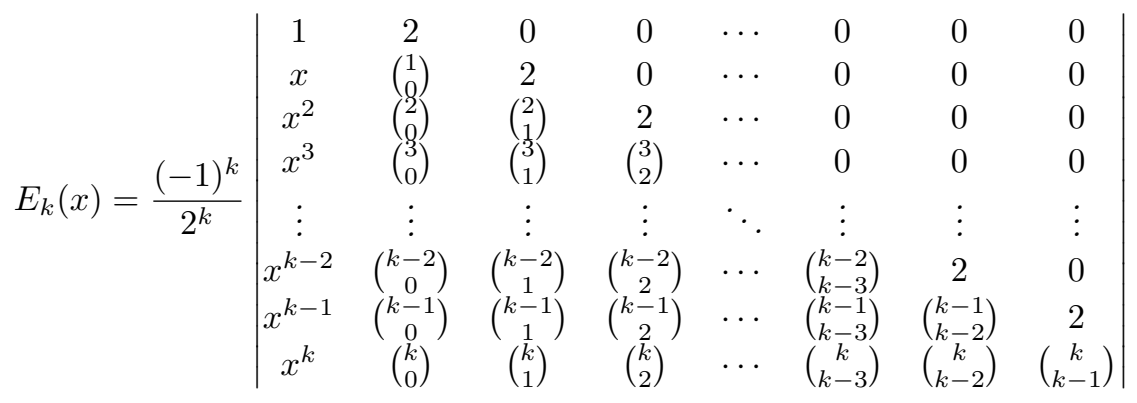

and, consequently, the Euler numbers $E_{k}$ can be expressed as

$$
E_{k}=(-1)^{k}\left|\begin{array}{cccccccc}
1 & 2 & 0 & 0 & \cdots & 0 & 0 & 0 \\
\frac{1}{2} & \left(\begin{array}{c}
1 \\
0
\end{array}\right) & 2 & 0 & \cdots & 0 & 0 & 0 \\
\frac{1}{2^{2}} & \left(\begin{array}{l}
2 \\
0
\end{array}\right) & \left(\begin{array}{l}
2 \\
1
\end{array}\right) & 2 & \cdots & 0 & 0 & 0 \\
\frac{1}{2^{3}} & \left(\begin{array}{l}
3 \\
0
\end{array}\right) & \left(\begin{array}{l}
3 \\
1
\end{array}\right) & \left(\begin{array}{l}
3 \\
2
\end{array}\right) & \cdots & 0 & 0 & 0 \\
\vdots & \vdots & \vdots & \vdots & \ddots & \vdots & \vdots & \vdots \\
\frac{1}{2^{k-2}} & \left(\begin{array}{c}
k-2 \\
0
\end{array}\right) & \left(\begin{array}{c}
k-2 \\
1
\end{array}\right) & \left(\begin{array}{c}
k-2 \\
2
\end{array}\right) & \cdots & \left(\begin{array}{c}
k-2 \\
k-3
\end{array}\right) & 2 & 0 \\
\frac{1}{2^{k-1}} & \left(\begin{array}{c}
k-1 \\
0
\end{array}\right) & \left(\begin{array}{c}
k-1 \\
1
\end{array}\right) & \left(\begin{array}{c}
k-1 \\
2
\end{array}\right) & \cdots & \left(\begin{array}{c}
k-1 \\
k-3
\end{array}\right) & \left(\begin{array}{c}
k-1 \\
k-2
\end{array}\right) & 2 \\
\frac{1}{2} & \left(\begin{array}{c}
k \\
2^{k}
\end{array}\right) & \left(\begin{array}{c}
k \\
1
\end{array}\right) & \left(\begin{array}{c}
k \\
2
\end{array}\right) & \cdots & \left(\begin{array}{c}
k \\
k-3
\end{array}\right) & \left(\begin{array}{c}
k \\
k-2
\end{array}\right) & \left(\begin{array}{c}
k \\
k-1
\end{array}\right)
\end{array}\right| .
$$

As an application of the expression (4), the following recurrence relation for the Euler polynomials and numbers $E_{k}(x)$ and $E_{k}$ can be derived. 
Theorem 2. The Euler polynomials and numbers $E_{k}(x)$ and $E_{k}$ satisfy the recurrence relations

$$
E_{k}(x)=-\frac{1}{2} \sum_{\ell=0}^{k-1}\left(\begin{array}{l}
k \\
\ell
\end{array}\right) E_{\ell}(x)+x^{k}
$$

and, consequently,

$$
E_{k}=-2^{k-1} \sum_{\ell=0}^{k-1} \frac{1}{2^{\ell}}\left(\begin{array}{l}
k \\
\ell
\end{array}\right) E_{\ell}+1 .
$$

\section{Proofs of Theorems 1 And 2}

Now we start out to prove our main results.

Proof of Theorem 1. In [6. Section 2.2, p. 849], 7, p. 94], and [8, Lemma 2.1], Exercise 5) in [1, p. 40] was reformulated as the following conclusion. Let $u(x)$ and $v(x) \neq 0$ be two differentiable functions. Let $U_{(n+1) \times 1}(x)$ be an $(n+1) \times 1$ matrix whose elements $u_{k, 1}(x)=u^{(k-1)}(x)$ for $1 \leq k \leq n+1$, let $V_{(n+1) \times n}(x)$ be an $(n+1) \times n$ matrix whose elements

$$
v_{i, j}(x)= \begin{cases}\left(\begin{array}{c}
i-1 \\
j-1
\end{array}\right) v^{(i-j)}(x), & i-j \geq 0 \\
0, & i-j<0\end{cases}
$$

for $1 \leq i \leq n+1$ and $1 \leq j \leq n$, and let $\left|W_{(n+1) \times(n+1)}(x)\right|$ denote the determinant of the $(n+1) \times(n+1)$ matrix

$$
W_{(n+1) \times(n+1)}(x)=\left(U_{(n+1) \times 1}(x) \quad V_{(n+1) \times n}(x)\right) .
$$

Then the $n$th derivative of the ratio $\frac{u(x)}{v(x)}$ can be computed by

$$
\frac{\mathrm{d}^{n}}{\mathrm{~d} x^{n}}\left[\frac{u(x)}{v(x)}\right]=(-1)^{n} \frac{\left|W_{(n+1) \times(n+1)}(x)\right|}{v^{n+1}(x)} .
$$

Let $u(t)=e^{x t}$ and $v(t)=e^{t}+1$. Then, by virtue of the formula (8),

$$
\frac{\mathrm{d}^{k}}{\mathrm{~d} t^{k}}\left(\frac{e^{x t}}{e^{t}+1}\right)=\frac{(-1)^{k}}{\left(e^{t}+1\right)^{k+1}}
$$


AN EXPRESSION AND A RECURRENCE FOR EULER POLYNOMIALS

$$
\begin{aligned}
& \times\left|\begin{array}{ccccccc}
e^{x t} & e^{t}+1 & 0 & \cdots & 0 & 0 & 0 \\
x e^{x t} & \left(\begin{array}{l}
1 \\
0
\end{array}\right) e^{t} & e^{t}+1 & \cdots & 0 & 0 & 0 \\
x^{2} e^{x t} & \left(\begin{array}{l}
2 \\
0
\end{array}\right) e^{t} & \left(\begin{array}{c}
2 \\
1
\end{array}\right) e^{t} & \cdots & 0 & 0 & 0 \\
x^{3} e^{x t} & \left(\begin{array}{c}
3 \\
0
\end{array}\right) e^{t} & \left(\begin{array}{l}
3 \\
1
\end{array}\right) e^{t} & \cdots & 0 & 0 & 0 \\
\vdots & \vdots & \vdots & \ddots & \vdots & \vdots & \vdots \\
x^{k-2} e^{x t} & \left(\begin{array}{c}
k-2 \\
0
\end{array}\right) e^{t} & \left(\begin{array}{c}
k-2 \\
1
\end{array}\right) e^{t} & \cdots & \left(\begin{array}{c}
k-2 \\
k-3
\end{array}\right) e^{t} & e^{t}+1 & 0 \\
x^{k-1} e^{x t} & \left(\begin{array}{c}
k-1 \\
0
\end{array}\right) e^{t} & \left(\begin{array}{c}
k-1 \\
1
\end{array}\right) e^{t} & \cdots & \left(\begin{array}{c}
k-1 \\
k-3
\end{array}\right) e^{t} & \left(\begin{array}{c}
k-1 \\
k-2
\end{array}\right) e^{t} & e^{t}+1 \\
k & 1 \\
x^{k} e^{x t} & \left(\begin{array}{c}
k \\
0
\end{array}\right) e^{t} & \left(\begin{array}{c}
k \\
1
\end{array}\right) e^{t} & \cdots & \left(\begin{array}{c}
k \\
k-3
\end{array}\right) e^{t} & \left(\begin{array}{c}
k \\
k-2
\end{array}\right) e^{t} & \left(\begin{array}{c}
k \\
k-1
\end{array}\right) e^{t}
\end{array}\right| \\
& \rightarrow \frac{(-1)^{k}}{2^{k+1}}\left|\begin{array}{cccccccc}
1 & 2 & 0 & 0 & \cdots & 0 & 0 & 0 \\
x & \left(\begin{array}{c}
1 \\
0
\end{array}\right) & 2 & 0 & \cdots & 0 & 0 & 0 \\
x^{2} & \left(\begin{array}{l}
2 \\
0
\end{array}\right) & \left(\begin{array}{l}
2 \\
1
\end{array}\right) & 2 & \cdots & 0 & 0 & 0 \\
x^{3} & \left(\begin{array}{c}
3 \\
0
\end{array}\right) & \left(\begin{array}{c}
3 \\
1
\end{array}\right) & \left(\begin{array}{c}
3 \\
2
\end{array}\right) & \cdots & 0 & 0 & 0 \\
\vdots & \vdots & \vdots & \vdots & \ddots & \vdots & \vdots & \vdots \\
x^{k-2} & \left(\begin{array}{c}
k-2 \\
0
\end{array}\right) & \left(\begin{array}{c}
k-2 \\
1
\end{array}\right) & \left(\begin{array}{c}
k-2 \\
2
\end{array}\right) & \cdots & \left(\begin{array}{c}
k-2 \\
k-3
\end{array}\right) & 2 & 0 \\
x^{k-1} & \left(\begin{array}{c}
k-1 \\
0
\end{array}\right) & \left(\begin{array}{c}
k-1 \\
1
\end{array}\right) & \left(\begin{array}{c}
k-1 \\
2
\end{array}\right) & \cdots & \left(\begin{array}{c}
k-1 \\
k-3
\end{array}\right) & \left(\begin{array}{c}
k-1 \\
k-2
\end{array}\right) & 2 \\
x^{k} & \left(\begin{array}{c}
k \\
k
\end{array}\right) & \left(\begin{array}{c}
k \\
1
\end{array}\right) & \left(\begin{array}{c}
k \\
2
\end{array}\right) & \cdots & \left(\begin{array}{c}
k \\
k-3
\end{array}\right) & \left(\begin{array}{c}
k \\
k-2
\end{array}\right) & \left(\begin{array}{c}
k \\
k-1
\end{array}\right)
\end{array}\right|
\end{aligned}
$$

as $t \rightarrow 0$ for $k \geq 0$. By the equation (2), the formula (4) is thus proved.

Considering the relation (3) and taking $x=\frac{1}{2}$ in (4) lead to the formula (5) readily. The proof of Theorem 1 is complete.

Proof of Theorem 2, Let

$$
D_{k+1}(x)=\left|\begin{array}{cccccccc}
1 & 2 & 0 & 0 & \cdots & 0 & 0 & 0 \\
x & \left(\begin{array}{c}
1 \\
0
\end{array}\right) & 2 & 0 & \cdots & 0 & 0 & 0 \\
x^{2} & \left(\begin{array}{l}
2 \\
0
\end{array}\right) & \left(\begin{array}{l}
2 \\
1
\end{array}\right) & 2 & \cdots & 0 & 0 & 0 \\
x^{3} & \left(\begin{array}{l}
3 \\
0
\end{array}\right) & \left(\begin{array}{c}
3 \\
1
\end{array}\right) & \left(\begin{array}{l}
3 \\
2
\end{array}\right) & \cdots & 0 & 0 & 0 \\
\vdots & \vdots & \vdots & \vdots & \ddots & \vdots & \vdots & \vdots \\
x^{k-2} & \left(\begin{array}{c}
k-2 \\
0
\end{array}\right) & \left(\begin{array}{c}
k-2 \\
1
\end{array}\right) & \left(\begin{array}{c}
k-2 \\
2
\end{array}\right) & \cdots & \left(\begin{array}{c}
k-2 \\
k-3
\end{array}\right) & 2 & 0 \\
x^{k-1} & \left(\begin{array}{c}
0 \\
0
\end{array}\right) & \left(\begin{array}{c}
k-1 \\
1
\end{array}\right) & \left(\begin{array}{c}
k-1 \\
2
\end{array}\right) & \cdots & \left(\begin{array}{c}
k-1 \\
k-3
\end{array}\right) & \left(\begin{array}{c}
k-1 \\
k-2
\end{array}\right) & 2 \\
x^{k} & \left(\begin{array}{c}
k \\
k
\end{array}\right) & \left(\begin{array}{c}
k \\
1
\end{array}\right) & \left(\begin{array}{c}
k \\
2
\end{array}\right) & \cdots & \left(\begin{array}{c}
k \\
k-3
\end{array}\right) & \left(\begin{array}{c}
k \\
k-2
\end{array}\right) & \left(\begin{array}{c}
k-1 \\
k-3
\end{array}\right)
\end{array}\right|
$$

for $k \geq 0$. Expanding $D_{k+1}(x)$ according to the last column consecutively and inductively reveals

$$
D_{k+1}(x)=\left(\begin{array}{c}
k \\
k-1
\end{array}\right)\left|\begin{array}{ccccccc}
1 & 2 & 0 & 0 & \cdots & 0 & 0 \\
x & \left(\begin{array}{c}
1 \\
0
\end{array}\right) & 2 & 0 & \cdots & 0 & 0 \\
x^{2} & \left(\begin{array}{c}
2 \\
0
\end{array}\right) & \left(\begin{array}{c}
2 \\
1
\end{array}\right) & 2 & \cdots & 0 & 0 \\
x^{3} & \left(\begin{array}{c}
3 \\
0
\end{array}\right) & \left(\begin{array}{c}
3 \\
1
\end{array}\right) & \left(\begin{array}{c}
3 \\
2
\end{array}\right) & \cdots & 0 & 0 \\
\vdots & \vdots & \vdots & \vdots & \ddots & \vdots & \vdots \\
x^{k-2} & \left(\begin{array}{c}
k-2 \\
0
\end{array}\right) & \left(\begin{array}{c}
k-2 \\
1
\end{array}\right) & \left(\begin{array}{c}
k-2 \\
2
\end{array}\right) & \cdots & \left(\begin{array}{c}
k-2 \\
k-3
\end{array}\right) & 2 \\
x^{k-1} & \left(\begin{array}{c}
k-1 \\
0
\end{array}\right) & \left(\begin{array}{c}
k-1 \\
1
\end{array}\right) & \left(\begin{array}{c}
k-1 \\
2
\end{array}\right) & \cdots & \left(\begin{array}{c}
k-1 \\
k-3
\end{array}\right) & \left(\begin{array}{l}
k-1 \\
k-2
\end{array}\right)
\end{array}\right|
$$


F. QI AND B.-N. GUO

$$
\begin{aligned}
& -2\left|\begin{array}{ccccccc}
1 & 2 & 0 & 0 & \cdots & 0 & 0 \\
x & \left(\begin{array}{c}
1 \\
0
\end{array}\right) & 2 & 0 & \cdots & 0 & 0 \\
x^{2} & \left(\begin{array}{c}
2 \\
0
\end{array}\right) & \left(\begin{array}{l}
2 \\
1
\end{array}\right) & 2 & \cdots & 0 & 0 \\
x^{3} & \left(\begin{array}{c}
3 \\
0
\end{array}\right) & \left(\begin{array}{l}
3 \\
1
\end{array}\right) & \left(\begin{array}{c}
3 \\
2
\end{array}\right) & \cdots & 0 & 0 \\
\vdots & \vdots & \vdots & \vdots & \ddots & \vdots & \vdots \\
x^{k-2} & \left(\begin{array}{c}
k-2 \\
0
\end{array}\right) & \left(\begin{array}{c}
k-2 \\
1
\end{array}\right) & \left(\begin{array}{c}
k-2 \\
2
\end{array}\right) & \cdots & \left(\begin{array}{c}
k-2 \\
k-3
\end{array}\right) & 2 \\
x^{k} & \left(\begin{array}{c}
k \\
0
\end{array}\right) & \left(\begin{array}{c}
k \\
1
\end{array}\right) & \left(\begin{array}{c}
k \\
2
\end{array}\right) & \cdots & \left(\begin{array}{c}
k \\
k-3
\end{array}\right) & \left(\begin{array}{c}
k \\
k-2
\end{array}\right)
\end{array}\right| \\
& =\left(\begin{array}{c}
k \\
k-1
\end{array}\right)\left|\begin{array}{ccccccc}
1 & 2 & 0 & 0 & \cdots & 0 & 0 \\
x & \left(\begin{array}{c}
1 \\
0
\end{array}\right) & 2 & 0 & \cdots & 0 & 0 \\
x^{2} & \left(\begin{array}{c}
2 \\
0
\end{array}\right) & \left(\begin{array}{l}
2 \\
1
\end{array}\right) & 2 & \cdots & 0 & 0 \\
x^{3} & \left(\begin{array}{c}
3 \\
0
\end{array}\right) & \left(\begin{array}{c}
3 \\
1
\end{array}\right) & \left(\begin{array}{c}
3 \\
2
\end{array}\right) & \cdots & 0 & 0 \\
\vdots & \vdots & \vdots & \vdots & \ddots & \vdots & \vdots \\
x^{k-2} & \left(\begin{array}{c}
k-2 \\
0
\end{array}\right) & \left(\begin{array}{c}
k-2 \\
1
\end{array}\right) & \left(\begin{array}{c}
k-2 \\
2
\end{array}\right) & \cdots & \left(\begin{array}{c}
k-2 \\
k-3
\end{array}\right) & 2 \\
x^{k-1} & \left(\begin{array}{c}
k-1 \\
0
\end{array}\right) & \left(\begin{array}{c}
k-1 \\
1
\end{array}\right) & \left(\begin{array}{c}
k-1 \\
2
\end{array}\right) & \cdots & \left(\begin{array}{c}
k-1 \\
k-3
\end{array}\right) & \left(\begin{array}{c}
k-2 \\
k-2
\end{array}\right.
\end{array}\right| \\
& -2\left(\begin{array}{c}
k \\
k-2
\end{array}\right)\left|\begin{array}{ccccccc}
1 & 2 & 0 & 0 & \cdots & 0 & 0 \\
x & \left(\begin{array}{c}
1 \\
0
\end{array}\right) & 2 & 0 & \cdots & 0 & 0 \\
x^{2} & \left(\begin{array}{l}
2 \\
0
\end{array}\right) & \left(\begin{array}{c}
2 \\
1
\end{array}\right) & 2 & \cdots & 0 & 0 \\
x^{3} & \left(\begin{array}{c}
3 \\
0
\end{array}\right) & \left(\begin{array}{c}
3 \\
1
\end{array}\right) & \left(\begin{array}{c}
3 \\
2
\end{array}\right) & \cdots & 0 & 0 \\
\vdots & \vdots & \vdots & \vdots & \ddots & \vdots & \vdots \\
x^{k-3} & \left(\begin{array}{c}
k-3 \\
0
\end{array}\right) & \left(\begin{array}{c}
k-3 \\
1
\end{array}\right) & \left(\begin{array}{c}
k-3 \\
2
\end{array}\right) & \cdots & \left(\begin{array}{c}
k-3 \\
k-4
\end{array}\right) & 2 \\
x^{k-2} & \left(\begin{array}{c}
k-2 \\
0
\end{array}\right) & \left(\begin{array}{c}
k-2 \\
1
\end{array}\right) & \left(\begin{array}{c}
k-2 \\
2
\end{array}\right) & \cdots & \left(\begin{array}{c}
k-2 \\
k-4
\end{array}\right) & k-3
\end{array}\right| \\
& +2^{2}\left(\begin{array}{c}
k \\
k-3
\end{array}\right)\left|\begin{array}{ccccccc}
1 & 2 & 0 & 0 & \cdots & 0 & 0 \\
x & \left(\begin{array}{l}
1 \\
0
\end{array}\right) & 2 & 0 & \cdots & 0 & 0 \\
x^{2} & \left(\begin{array}{l}
2 \\
0
\end{array}\right) & \left(\begin{array}{l}
2 \\
1
\end{array}\right) & 2 & \cdots & 0 & 0 \\
x^{3} & \left(\begin{array}{c}
3 \\
0
\end{array}\right) & \left(\begin{array}{c}
3 \\
1
\end{array}\right) & \left(\begin{array}{c}
3 \\
2
\end{array}\right) & \cdots & 0 & 0 \\
\vdots & \vdots & \vdots & \vdots & \ddots & \vdots & \vdots \\
x^{k-4} & \left(\begin{array}{c}
k-4 \\
0
\end{array}\right) & \left(\begin{array}{c}
k-4 \\
1
\end{array}\right) & \left(\begin{array}{c}
k-4 \\
2
\end{array}\right) & \cdots & \left(\begin{array}{c}
k-4 \\
k-5
\end{array}\right) & 2 \\
x^{k-3} & \left(\begin{array}{c}
k-3 \\
0
\end{array}\right) & \left(\begin{array}{c}
k-3 \\
1
\end{array}\right) & \left(\begin{array}{c}
k-3 \\
2
\end{array}\right) & \cdots & \left(\begin{array}{c}
k-3 \\
k-5
\end{array}\right) & \left(\begin{array}{c}
k-3 \\
k-4
\end{array}\right)
\end{array}\right| \\
& -2^{3}\left|\begin{array}{ccccccc}
1 & 2 & 0 & 0 & \cdots & 0 & 0 \\
x & \left(\begin{array}{c}
1 \\
0
\end{array}\right) & 2 & 0 & \cdots & 0 & 0 \\
x^{2} & \left(\begin{array}{l}
2 \\
0
\end{array}\right) & \left(\begin{array}{l}
2 \\
1
\end{array}\right) & 2 & \cdots & 0 & 0 \\
x^{3} & \left(\begin{array}{l}
3 \\
0
\end{array}\right) & \left(\begin{array}{l}
3 \\
1
\end{array}\right) & \left(\begin{array}{l}
3 \\
2
\end{array}\right) & \cdots & 0 & 0 \\
\vdots & \vdots & \vdots & \vdots & \ddots & \vdots & \vdots \\
x^{k-4} & \left(\begin{array}{c}
k-4 \\
0
\end{array}\right) & \left(\begin{array}{c}
k-4 \\
1
\end{array}\right) & \left(\begin{array}{c}
k-4 \\
2
\end{array}\right) & \cdots & \left(\begin{array}{c}
k-4 \\
k-5 \\
k
\end{array}\right) & 2 \\
x^{k} & \left(\begin{array}{c}
k \\
0
\end{array}\right) & \left(\begin{array}{c}
k \\
1
\end{array}\right) & \left(\begin{array}{c}
k \\
2
\end{array}\right) & \cdots & \left(\begin{array}{c}
k \\
k-5
\end{array}\right) & \left(\begin{array}{c}
k-4 \\
k-4
\end{array}\right)
\end{array}\right| \\
& =\sum_{\ell=0}^{m}(-1)^{\ell} 2^{\ell}\left(\begin{array}{c}
k \\
k-\ell-1
\end{array}\right) D_{k-\ell}(x)+(-1)^{m+1} 2^{m+1}
\end{aligned}
$$




$$
\begin{aligned}
& \begin{array}{cccccc}
1 & 2 & 0 & \cdots & 0 & 0 \\
x & \left(\begin{array}{c}
1 \\
0
\end{array}\right) & 2 & \cdots & 0 & 0 \\
x^{2} & \left(\begin{array}{c}
2 \\
0
\end{array}\right) & \left(\begin{array}{l}
2 \\
1
\end{array}\right) & \cdots & 0 & 0 \\
x^{3} & \left(\begin{array}{l}
3 \\
0
\end{array}\right) & \left(\begin{array}{l}
3 \\
1
\end{array}\right) & \cdots & 0 & 0 \\
\vdots & \vdots & \vdots & & \ddots & \vdots \\
x^{k-m-2} & \left(\begin{array}{c}
k-m-2 \\
0
\end{array}\right) & \left(\begin{array}{c}
k-m-2 \\
1
\end{array}\right) & \cdots & \left(\begin{array}{c}
k-m-2 \\
k-m-3
\end{array}\right) & 2 \\
k & k \\
x^{k} & \left(\begin{array}{c}
k \\
0
\end{array}\right) & \left(\begin{array}{c}
k \\
1
\end{array}\right) & \cdots & \left(\begin{array}{c}
k \\
k-m-2
\end{array}\right)
\end{array} \mid \\
= & \sum_{\ell=0}^{k-2}(-1)^{\ell} 2^{\ell}\left(\begin{array}{c}
k \\
k-\ell-1
\end{array}\right) D_{k-\ell}(x)+(-1)^{k-1} 2^{k-1}\left|\begin{array}{cc}
1 & 2 \\
x^{k} & \left(\begin{array}{c}
k \\
0
\end{array}\right)
\end{array}\right| \\
= & \sum_{\ell=0}^{k-2}(-1)^{\ell} 2^{\ell}\left(\begin{array}{c}
k \\
k-\ell-1
\end{array}\right) D_{k-\ell}(x)+(-1)^{k-1} 2^{k-1}\left[\left(\begin{array}{c}
k \\
0
\end{array}\right)-2 x^{k}\right] \\
= & \sum_{\ell=0}^{k-1}(-1)^{\ell} 2^{\ell}\left(\begin{array}{c}
k \\
k-\ell-1
\end{array}\right) D_{k-\ell}(x)+(-1)^{k} 2^{k} x^{k} \\
= & \sum_{\ell=0}^{k-1}(-1)^{\ell} 2^{\ell}\left(\begin{array}{c}
k \\
k-\ell-1
\end{array}\right) \frac{2^{k-\ell-1}}{(-1)^{k-\ell-1}}\left[\frac{(-1)^{k-\ell-1}}{2^{k-\ell-1}} D_{k-\ell}(x)\right]+(-1)^{k} 2^{k} x^{k} \\
= & (-1)^{k-1} 2^{k-1} \sum_{\ell=0}^{k-1}\left(\begin{array}{c}
k \\
k-\ell-1
\end{array}\right) E_{k-\ell-1}(x)+(-1)^{k} 2^{k} x^{k} .
\end{aligned}
$$

Accordingly, it follows that

$$
\begin{aligned}
E_{k}(x)=\frac{(-1)^{k}}{2^{k}} D_{k+1}(x) & =-\frac{1}{2} \sum_{\ell=0}^{k-1}\left(\begin{array}{c}
k \\
k-\ell-1
\end{array}\right) E_{k-\ell-1}(x)+x^{k} \\
& =-\frac{1}{2} \sum_{\ell=0}^{k-1}\left(\begin{array}{l}
k \\
\ell
\end{array}\right) E_{\ell}(x)+x^{k} .
\end{aligned}
$$

The relation (6) is thus proved.

Taking $x=\frac{1}{2}$ in (6) and making use of the relation (3) result in the relation (7). The proof of Theorem 2 is complete.

Remark 1 . The expression (5) can also be obtained by applying the formula (8) to the functions $u(t)=e^{t / 2}$ and $v(t)=e^{t}+1$ and considering the generating function $\frac{2 e^{t / 2}}{e^{t}+1}$ in the equation (1).

\section{REFERENCES}

[1] N. Bourbaki, Elements of Mathematics: Functions of a Real Variable: Elementary Theory, Translated from the 1976 French original by Philip Spain. Elements of Mathematics (Berlin). Springer-Verlag, Berlin, 2004; Available online at http://dx.doi.org/10.1007/ 978-3-642-59315-4

[2] B.-N. Guo and F. Qi, Explicit formulae for computing Euler polynomials in terms of Stirling numbers of the second kind, J. Comput. Appl. Math. 272 (2014), 251-257; Available online at http://dx.doi.org/10.1016/j.cam.2014.05.018

[3] B.-N. Guo and F. Qi, Explicit formulas for special values of the Bell polynomials of the second kind and the Euler numbers, ResearchGate Technical Report (2015), available online at http://dx.doi.org/10.13140/2.1.3794.8808 
[4] G. A. Korn and T. M. Korn, Mathematical Handbook for Scientists and Engineers, Second, enlarged and revised edition, McGraw-Hill Book Co., New York-Toronto, Ont.-London, 1968. (in Russian)

[5] J. Malenfant, Finite, closed-form expressions for the partition function and for Euler, Bernoulli, and Stirling numbers, arXiv preprint (2011), available online at http://arxiv. org/abs/1103.1585v6

[6] F. Qi, Derivatives of tangent function and tangent numbers, Appl. Math. Comput. 268 (2015), 844-858; Available online at http://dx.doi.org/10.1016/j.amc.2015.06.123

[7] F. Qi and R. J. Chapman, Two closed forms for the Bernoulli polynomials, J. Number Theory 159 (2016), 89-100; Available online at http://dx.doi.org/10.1016/j.jnt.2015.07.021

[8] C.-F. Wei and F. Qi, Several closed expressions for the Euler numbers, J. Inequal. Appl. 2015, 2015:219, 8 pages; Available online at http://dx.doi.org/10.1186/s13660-015-0738-9

[9] WikiPedia, Euler number, From the Free Encyclopedia; Available online at https://en. wikipedia.org/wiki/Euler_number (Accessed on 9 December 2015)

$U R L:$ https://qifeng618.wordpress.com

$U R L:$ http://www.researchgate.net/profile/Bai-Ni_Guo/ 